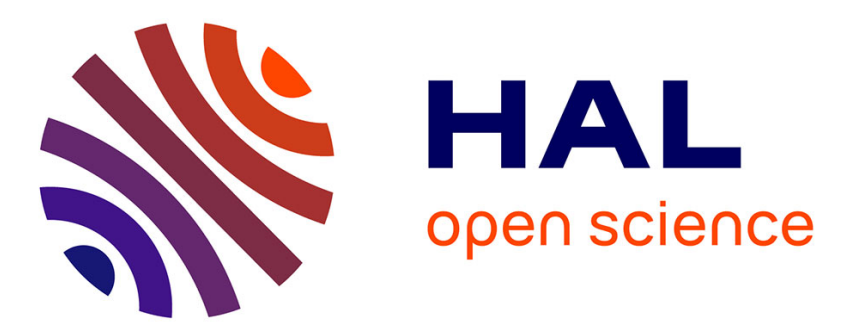

\title{
Improved Coprime Linear Array Configuration for Moving Platform in DOA Estimation
}

Xiao Yang, Yide Wang, Pascal Chargé

\section{To cite this version:}

Xiao Yang, Yide Wang, Pascal Chargé. Improved Coprime Linear Array Configuration for Moving Platform in DOA Estimation. IEEE Communications Letters, 2021, 25 (2), pp.470-473. 10.1109/LCOMM.2020.3029322 . hal-02958672

\section{HAL Id: hal-02958672 \\ https://hal.science/hal-02958672}

Submitted on 17 Feb 2021

HAL is a multi-disciplinary open access archive for the deposit and dissemination of scientific research documents, whether they are published or not. The documents may come from teaching and research institutions in France or abroad, or from public or private research centers.
L'archive ouverte pluridisciplinaire HAL, est destinée au dépôt et à la diffusion de documents scientifiques de niveau recherche, publiés ou non, émanant des établissements d'enseignement et de recherche français ou étrangers, des laboratoires publics ou privés. 


\title{
Improved Coprime Linear Array Configuration for Moving Platform in DOA Estimation
}

\author{
Xiao Yang, Yide Wang, and Pascal Chargé
}

\begin{abstract}
Moving platform based coprime linear arrays (CLAs) have been studied in recent years. It is shown that by shifting a CLA a half wavelength, the resulting difference coarray can be regarded as the union of the difference coarray of the original CLA and its two shifted versions with one lag to the left and one lag to the right, respectively, filling the majority or all holes in the difference coarray. However, only the lags which are neighbors of holes are actually useful, while the shifts of the other lags generate lags which already exist, contributing then negligibly to the increase of the degrees of freedom (DOFs). In this letter, an improved CLA configuration for moving platform is proposed. By judiciously designing the sensor positions, all lags can be utilized to fill holes, consequently a difference coarray with more consecutive lags can be obtained. Compared with the original configuration, the proposed configuration can detect more sources with the same number of sensors and same length of array motion.
\end{abstract}

Index Terms-array motion, coprime linear array, difference coarray, DOA estimation, high DOFs

\section{INTRODUCTION}

D IRECTION of arrival (DOA) estimation with high degrees of freedom (DOFs) has attracted lots of attention in wireless communication system. With precise DOA information, transmission energy can be focalized to the wanted users and interferences can be efficiently eliminated to increase the channel capacity, and high DOFs can increase the number of users served [1], [2]. Consisting of two uniform linear subarrays with large inter-element spacing, a coprime linear array (CLA) can resolve $O(M N)$ sources with $O(M+N)$ sensors by exploiting the difference coarray obtained from the covariance matrix of the received signals [3]. Being superior to the conventional uniform linear arrays (ULAs), which can only detect up to $M-1$ sources with $M$ sensors, CLAs have been considered as a promising array configuration thanks to its great advantage in terms of DOFs [4], [5].

However, because of the existence of holes in the difference coarray of a CLA and only the consecutive lags can be directly used to implement DOA estimation by subspace based algorithms like MUSIC [6] and ESPRIT [7], the effective DOFs, which roughly equal to the half number of the consecutive lags, are not as high as expected. To solve this problem, many methods have been proposed to fill the holes and lengthen the consecutive part of the difference coarray. In [8], the CLAs are generalized with two operations, by which two improved CLA configurations, namely coprime arrays with compressed interelement spacing (CACIS) and coprime arrays with displaced subarrays (CADiS), are proposed. By selecting appropriate

This work is supported by China Scholarship Council (File No.201708440322).

The authors are with the Institut d'Électronique et de Télécommunications de Rennes UMR CNRS 6164, Poltytech de Nantes, 44306 Nantes, France (e-mail:xiao.yang1@etu.univ-nantes.fr). parameters, a difference coarray with a longer consecutive part can be obtained, and the effective DOFs are increased consequently. In [9], the holes in the difference coarray are interpolated by nuclear norm minimization, allowing to use the remaining elements of the difference coarray and increase the number of detectable signals. In [10], a new coprime configuration named $k$-times extended coprime array with an additional complementary subarray is proposed, which exhibits a complete difference coarray as well as higher DOFs.

The above mentioned methods consider the CLAs based on fixed platform, and CLAs can also be mounted on a moving platform, like air-borne, vehicle-attached or ship-based. In [11] and [12], a moving CLA is considered, and the shift of the CLA is set to the half wavelength of incoming signals such that the source positions, incoming directions and signal temporal properties can be assumed to be constant. It is shown that due to such array motion, the resulting difference coarray can be regarded as the union of the difference coarray of the original CLA and its two shifted versions with one lag to the left and one lag to the right, respectively, such that the neighboring holes of each lag can be filled, generating then a difference coarray with more consecutive lags and increasing the effective DOFs. However, only the lags which are neighbors of holes are utilized to fill the holes. For the lags in the consecutive part without neighboring holes, the shifts of them generate lags already existing in the difference coarray of the original CLA, contributing negligibly to the resulting difference coarray and the increase of the DOFs.

In this letter, an improved CLA configuration for moving platform is proposed. By judiciously designing the sensor element positions, a lengthened difference coarray with controled hole positions is obtained, such that all lags can be utilized to fill the holes by array motion. Contrary to [13] which discusses nested arrays, our proposition is dedicated to CLAs as [11] and [12]. The proposed CLA configuration has a difference coarray with much more consecutive lags and higher effective DOFs than [11] and [12] with the same number of sensors and same length of array shift. Simulation results are provided to show the performance of the proposition.

\section{SignAL MODEL}

Consider a CLA composed of two overlapped uniform linear subarrays, having $N$ and $M$ sensor elements, with interelement spacings $M d$ and $N d$, respectively. $M$ and $N$ are two coprime integers and $d=\frac{\lambda}{2}$ is the unit inter-element spacing with $\lambda$ the wavelength of incoming signals. Without loss of generality, it is assumed that $M<N$. The sensors are located at positions $\left\{d_{1}, d_{2}, \ldots, d_{L}\right\}$, where $L$ is the total number of sensors. Because the first sensor is shared by the two subarrays and set as the reference point, we have $L=M+N-1$ 


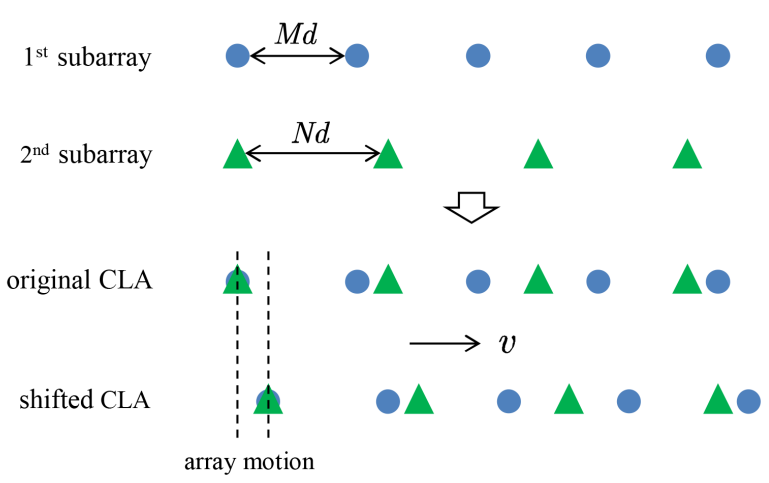

Fig. 1. Moving platform based CLA with $N=5$ and $M=4$.

and $d_{1}=0$. The CLA moves along the array direction with a constant velocity $v$. The DOAs of sources and signal temporal properties are assumed to be constant for a small array motion. Fig. 1 shows the situation where $N=5$ and $M=4$.

Suppose that there are $K$ ( $K$ is supposed to be known) uncorrelated, far-field and narrowband signals impinging on the CLA from directions $\left\{\theta_{1}, \theta_{2}, \ldots, \theta_{K}\right\}$, respectively, with $-90^{\circ}<\theta_{k}<90^{\circ}$ and $1 \leq k \leq K$. The observation of the CLA, at time $t$, can be expressed as [11], [12]

$$
\mathbf{x}(t)=\mathbf{A} \boldsymbol{\Phi} \mathbf{s}(t)+\mathbf{n}(t)
$$

where $\mathbf{A}=\left[\mathbf{a}\left(\theta_{1}\right), \mathbf{a}\left(\theta_{2}\right), \ldots, \mathbf{a}\left(\theta_{K}\right)\right]$ is the directional matrix of the CLA, with directional vector $\mathbf{a}\left(\theta_{k}\right)=\left[1, e^{j 2 \pi \frac{d_{2}}{\lambda} \sin \theta_{k}}, \ldots, e^{j 2 \pi \frac{d_{L}}{\lambda} \sin \theta_{k}}\right]^{T} ; \quad \mathbf{\Phi}=$ $\operatorname{diag}\left\{e^{j 2 \pi \frac{v t}{\lambda} \sin \theta_{1}}, e^{j 2 \pi \frac{v t}{\lambda} \sin \theta_{2}}, \ldots, e^{j 2 \pi \frac{v t}{\lambda} \sin \theta_{K}}\right\} \quad$ indicates the movement of the array; $\mathbf{s}(t)=\left[s_{1}(t), s_{2}(t), \ldots, s_{K}(t)\right]^{T}$ represents the incoming signal vector; $\mathbf{n}(t)$ denotes the white Gaussian noise vector with zero-mean and covariance matrix $\sigma^{2} \mathbf{I}_{L}$, with $\mathbf{I}_{L}$ the $L \times L$ identity matrix and $\sigma^{2}$ the noise power, and the noise is supposed to be independent from the source signals.

At time $t+\tau$, the observation of the CLA becomes

$$
\mathbf{x}(t+\tau)=\mathbf{B} \Phi \mathbf{s}(t+\tau)+\mathbf{n}(t+\tau)
$$

where $\mathbf{B}=\left[\mathbf{b}\left(\theta_{1}\right), \mathbf{b}\left(\theta_{2}\right), \ldots, \mathbf{b}\left(\theta_{K}\right)\right]$ and $\mathbf{b}\left(\theta_{k}\right)=$ $\left[e^{j 2 \pi \frac{v \tau}{\lambda} \sin \theta_{k}}, e^{j 2 \pi \frac{v \tau+d_{2}}{\lambda} \sin \theta_{k}}, \ldots, e^{j 2 \pi \frac{v \tau+d_{L}}{\lambda}} \sin \theta_{k}\right]^{T}$

denote the updated directional matrix and vector, respectively. By setting $v \tau=d=\frac{\lambda}{2}$, we have $\mathbf{b}\left(\theta_{k}\right)=\left[e^{j 2 \pi \frac{d}{\lambda} \sin \theta_{k}}, e^{j 2 \pi \frac{d+d_{2}}{\lambda} \sin \theta_{k}}, \ldots, e^{j 2 \pi \frac{d+d_{L}}{\lambda}} \sin \theta_{k}\right]^{T}$.

For narrowband signals with carrier frequency $f$, we have $s_{k}(t+\tau)=e^{j 2 \pi f \tau} s_{k}(t)$, and (2) can be re-written as

$$
\mathbf{x}(t+\tau)=e^{j 2 \pi f \tau} \mathbf{B} \boldsymbol{\Phi} \mathbf{s}(t)+\mathbf{n}(t+\tau)
$$

Then using the phase factor compensating technique described in [14], we get

$$
\tilde{\mathbf{x}}(t+\tau)=e^{-j 2 \pi f \tau} \mathbf{x}(t+\tau)=\mathbf{B} \boldsymbol{\Phi} \mathbf{s}(t)+\tilde{\mathbf{n}}(t+\tau)
$$

with $\tilde{\mathbf{n}}(t+\tau)=e^{-j 2 \pi f \tau} \mathbf{n}(t+\tau)$, and by combining (1) and (4), we obtain the following observation of the synthetic array:

$$
\mathbf{y}(t)=\left[\begin{array}{c}
\mathbf{x}(t) \\
\tilde{\mathbf{x}}(t+\tau)
\end{array}\right]=\mathbf{A}_{s} \mathbf{s}_{s}(t)+\left[\begin{array}{c}
\mathbf{n}(t) \\
\tilde{\mathbf{n}}(t+\tau)
\end{array}\right]
$$

where $\mathbf{A}_{s}=\left[\mathbf{A}^{T}, \mathbf{B}^{T}\right]^{T}$ stands for the directional matrix of the synthetic array, composed of the original CLA and the shifted CLA after moving for a unit inter-element spacing $d$, and $\mathbf{s}_{s}(t)=\boldsymbol{\Phi} \mathbf{s}(t)$ is the equivalent incoming signal vector.

\section{ANALYSIS OF THE DIFFERENCE COARRAY}

According to the CLA configuration introduced in Section II, taking $d$ as unit, the sensor positions sets of the original and shifted CLAs can be given as

$$
\begin{aligned}
\mathbb{P}_{o}= & \{M n, 0 \leq n \leq N-1\} \cup \\
& \{N m, 0 \leq m \leq M-1\} \\
\mathbb{P}_{s}= & \{M n+1,0 \leq n \leq N-1\} \cup \\
& \{N m+1,0 \leq m \leq M-1\}
\end{aligned}
$$

According to [11] and [12], since the synthetic array can be regarded as the union of the original and shifted CLAs, the corresponding difference coarray positions set is composed of the sets of cross-lags between all the subarrays of the original CLA and the shifted CLA, which can be expressed as

$$
\mathbb{D}=\mathbb{D}_{12} \cup \mathbb{D}_{11^{\prime}} \cup \mathbb{D}_{12^{\prime}} \cup \mathbb{D}_{21^{\prime}} \cup \mathbb{D}_{22^{\prime}} \cup \mathbb{D}_{1^{\prime} 2^{\prime}}
$$

where subscripts 1 and 2 refer to the $1^{\text {st }}$ and $2^{\text {nd }}$ subarrays of the original CLA, and $1^{\prime}$ and $2^{\prime}$ refer to the $1^{\text {st }}$ and $2^{\text {nd }}$ subarrays of the shifted CLA, respectively. The self-lags of them form subsets of the sets of cross-lags and do not affect the resulting difference coarray. $\mathbb{D}_{u w}\left(u, w \in\left\{1,2,1^{\prime}, 2^{\prime}\right\}\right)$, denoting the sets of the cross-lags between the subarrays specified by subscripts $u$ and $w$, can be expressed as

$$
\begin{aligned}
& \mathbb{D}_{12}=\left\{M k_{1}-N k_{2}\right\} \cup\left\{N k_{2}-M k_{1}\right\} \\
& \mathbb{D}_{11^{\prime}}=\left\{M k_{1}-M k_{1}^{\prime}-1\right\} \cup\left\{M k_{1}^{\prime}-M k_{1}+1\right\} \\
& \mathbb{D}_{12^{\prime}}=\left\{M k_{1}-N k_{2}^{\prime}-1\right\} \cup\left\{N k_{2}^{\prime}-M k_{1}+1\right\} \\
& \mathbb{D}_{21^{\prime}}=\left\{N k_{2}-M k_{1}^{\prime}-1\right\} \cup\left\{M k_{1}^{\prime}-N k_{2}+1\right\} \\
& \mathbb{D}_{22^{\prime}}=\left\{N k_{2}-N k_{2}^{\prime}-1\right\} \cup\left\{N k_{2}^{\prime}-N k_{2}+1\right\} \\
& \mathbb{D}_{1^{\prime} 2^{\prime}}=\left\{M k_{1}^{\prime}-N k_{2}^{\prime}\right\} \cup\left\{N k_{2}^{\prime}-M k_{1}^{\prime}\right\}
\end{aligned}
$$

where $k_{1}, k_{2}, k_{1}^{\prime}$ and $k_{2}^{\prime}$ are four integers with $0 \leq k_{1}, k_{1}^{\prime} \leq$ $N-1$ and $0 \leq k_{2}, k_{2}^{\prime} \leq M-1$.

It is proved in [11] and [12] that the resulting difference coarray after array motion (8) can be re-written as

$$
\mathbb{D}=\mathbb{D}_{12} \cup \mathbb{D}_{12}^{L} \cup \mathbb{D}_{12}^{R}
$$

with

$$
\begin{aligned}
& \mathbb{D}_{12}^{L}=\mathbb{D}_{12}-1 \\
& \mathbb{D}_{12}^{R}=\mathbb{D}_{12}+1
\end{aligned}
$$

which can be regarded as the difference coarray of the original CLA shifting one lag to the left and one lag to the right, respectively. Consequently, the neighboring holes of each lag in the difference coarray of the original CLA can be filled due to the array motion. For the same reason, two additional lags can be obtained at the two ends of the original difference coarray, lengthening the consecutive part and increasing the effective DOFs.

For the moving platform based CLA in Fig. 1, Fig. 2 and Fig. 3 show the difference coarrays of the CLA before and after motion, where blue dots and red rhombuses represent the lags and holes, respectively. It can be observed that being regarded as the lags of $\mathbb{D}_{12}$ shifting one lag to the left and one lag to the right, respectively, the lags of $\mathbb{D}_{12}^{L}$ and $\mathbb{D}_{12}^{R}$ fill the holes in $\mathbb{D}_{12}$, and a hole-free difference coarray is generated. Meanwhile, since $\mathbb{D}_{12}^{L}$ and $\mathbb{D}_{12}^{R}$ are the shifted versions of $\mathbb{D}_{12}$ with only one lag to the left and right, respectively, if there are 


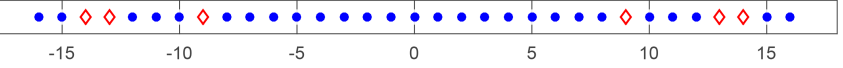

Fig. 2. Difference coarray of the CLA with $N=5$ and $M=4$ before array motion $\left(\mathbb{D}_{12}\right)$.

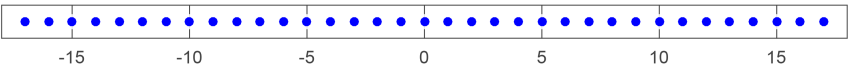

Fig. 3. Difference coarray of the CLA with $N=5$ and $M=4$ after array motion $\left(\mathbb{D}=\mathbb{D}_{12} \cup \mathbb{D}_{12}^{L} \cup \mathbb{D}_{12}^{R}\right)$.

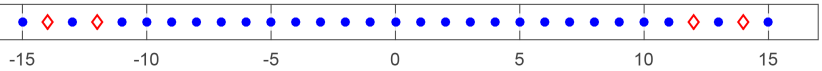

Fig. 4. Difference coarray of the inter-element spacing compressed CLA with $N=5$ and $M=4$

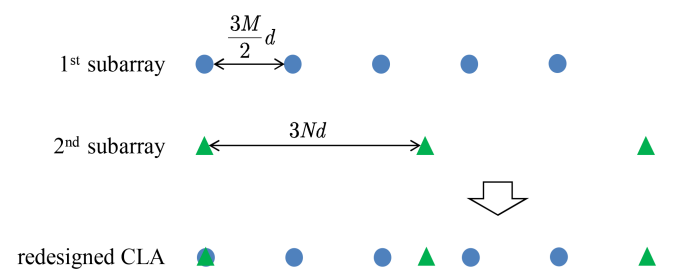

Fig. 5. Redesigned CLA with $N=5$ and $M=4$.

three or more consecutive holes in $\mathbb{D}_{12}$, only the holes having neighboring lags can be filled. For the other holes without neighboring lags, they would be remained unfilled because the corresponding positions in $\mathbb{D}_{12}^{L}$ and $\mathbb{D}_{12}^{R}$ are also holes.

Even if the resulting difference coarray after motion is not always hole-free, the number of consecutive lags increases significantly with array motion. The closed-form expression of the number of consecutive lags for different values of $M$ and $N$ is derived in [11], which is at most $2 M(N-1)+3$ when the resulting difference coarray after motion is hole-free.

However, it can be noticed that only the lags which are neighbors of holes (e.g. $\{x \mid x= \pm 8, \pm 10, \pm 12, \pm 15\}$ in Fig. 2) are used to fill the holes. For the consecutive lags without neighboring holes (e.g. $\{x \mid-7 \leq x \leq 7\}$ in Fig. 2 ), the shifts of them generate lags which already exist in the difference coarray of the original CLA before motion. Without generating new lags, such lags do not contribute to the increase of the DOFs by array motion. In the next section, an improved configuration of moving platform based CLA is proposed, in which all the lags can be utilized to fill holes by array motion, and more consecutive lags as well as higher effective DOFs can be obtained.

\section{PROposed CONFIGURATION}

Firstly, the inter-element spacing of the subarray with $N$ sensor elements is compressed from $M d$ to $\frac{M d}{2}$. The resulting difference coarray before array motion has a consecutive part with $M N+M-1$ lags in the range of $\left[-\frac{M N}{2}-\frac{M}{2}+1, \frac{M N}{2}+\frac{M}{2}-1\right]$.

Proof: see [8]
TABLE I

NUMBERS OF CONSECUTIVE LAGS COMPARISON

\begin{tabular}{|c|c|c|}
\hline \multirow{4}{*}{$\begin{array}{c}\text { original } \\
\text { configuration }\end{array}$} & $(M, N)$ & consecutive lags \\
\cline { 2 - 3 } & $(4,5)$ & 35 \\
\cline { 2 - 3 } & $(4,7)$ & 51 \\
\cline { 2 - 3 } & $(6,1)$ & 51 \\
\cline { 2 - 3 } & $(8,9)$ & 79 \\
\cline { 2 - 3 } & $(8,11)$ & 67 \\
\hline \multirow{4}{*}{$\begin{array}{c}\text { proposed } \\
\text { configuration }\end{array}$} & $(4,5)$ & 147 \\
\cline { 2 - 3 } & $(4,7)$ & 69 \\
\cline { 2 - 3 } & $(6,7)$ & 93 \\
\cline { 2 - 3 } & $(8,11)$ & 213 \\
\cline { 2 - 3 } & $(8,11)$ & 237 \\
\hline
\end{tabular}

Fig. 4 shows the resulting difference coarray after compressing the inter-element spacing of the CLA in Fig. 1. In order to use the lags in the consecutive part without neighboring holes by array motion, we propose to lengthen the inter-element spacings of both the subarrays three times, from $\frac{M d}{2}$ and $N d$ to $\frac{3 M d}{2}$ and $3 N d$, respectively. Consequently, the positions of the lags in the new difference coarray are also enlarged three times, such that in the range of $\left[-\frac{3 M N}{2}-\frac{3 M}{2}+3, \frac{3 M N}{2}+\frac{3 M}{2}-\right.$ $3]$, lags are uniformly distributed, and every two adjacent lags are exactly separated by two holes, which are generated by the lengthening of the inter-element spacing. Being different from the CLA configuration in Fig. 1 used in [11] and [12], the redesigned CLA with the same values of $N$ and $M$ and its corresponding difference coarray are shown in Fig. 5 and Fig. 6, respectively. It can be observed that for the lags in the range of $\left[-\frac{3 M N}{2}-\frac{3 M}{2}+3, \frac{3 M N}{2}+\frac{3 M}{2}-3\right]$, the shifts of them with one lag to the left and one lag to the right generate new lags which do not exist in the original difference coarray of the redesigned CLA, filling their neighboring holes and generating a consecutive part without holes in the range of $\left[-\frac{3 M N}{2}-\frac{3 M}{2}+2, \frac{3 M N}{2}+\frac{3 M}{2}-2\right]$. The resulting difference coarray after array motion is shown in Fig. 7.

\section{DOFs COMParison With Simulation REsults}

For the original moving platform based CLA configuration in [11] and [12], we can obtain a difference coarray with at most $2 M(N-1)+3$ consecutive lags after array motion. For the proposed configuration, although the obtained difference coarray is not complete, it has a longer consecutive part in the range of $\left[-\frac{3 M N}{2}-\frac{3 M}{2}+2, \frac{3 M N}{2}+\frac{3 M}{2}-2\right]$ with a total number of $3 M N+3 M-3$ consecutive lags, which is obviously more than that of the original configuration even when the latter is hole-free. Therefore, the proposed configuration can significantly increase the effective DOFs with the same number of sensor elements and same length of array motion. Table I shows the comparison of the numbers of consecutive lags in the final difference coarrays of the original and proposed CLA configurations with different values of $M$ and $N$.

To show the benefit of the proposed CLA configuration in terms of DOFs increasing, the MUSIC method is applied 


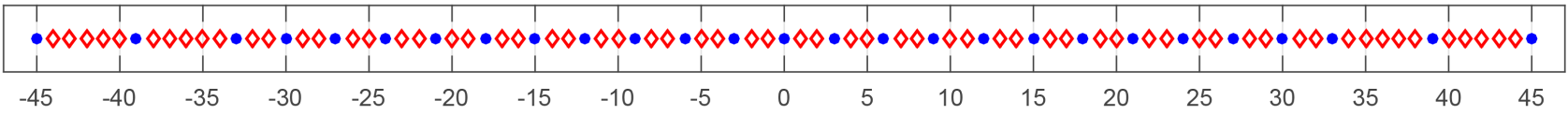

Fig. 6. Difference coarray of the redesigned CLA with $N=5$ and $M=4$ before array motion.

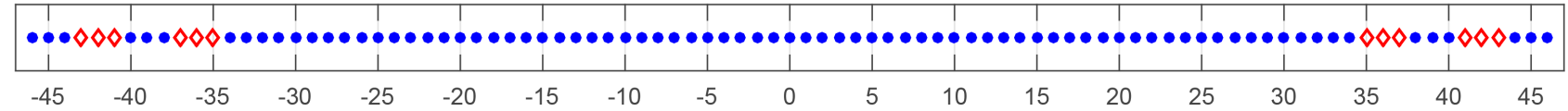

Fig. 7. Difference coarray of the redesigned CLA with $N=5$ and $M=4$ after array motion.

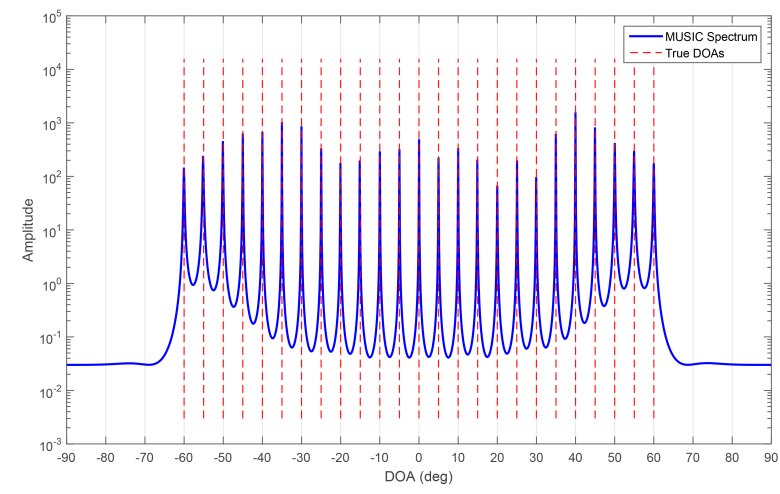

Fig. 8. MUSIC spectrum of the proposed CLA configuration with $N=5$ and $M=4$.

to perform DOA estimation on a CLA with $N=5$ and $M=4$. For the original CLA configuration in [11] and [12], a difference coarray with 35 consecutive lags is obtained after the array motion with a length of $\frac{\lambda}{2}$. After the step of spatial smoothing [3], a rank restored covariance matrix with dimension $18 \times 18$ is obtained, and up to 17 signals can be detected according to the principle of the MUSIC method. For the proposed configuration, a difference coarray with 69 consecutive lags is obtained after the same length of array motion, therefore a rank restored covariance matrix with dimension $35 \times 35$ is obtained, and up to 34 signals can be detected. Fig. 8 depicts the DOA estimation result of the proposed configuration with 25 sources uniformly distributed from $-60^{\circ}$ to $60^{\circ}$ with the signal-to-noise ratio (SNR) being $10 \mathrm{~dB}$ and the number of snapshots being 2000 . For the original CLA configuration in [11] and [12], because the number of sources exceeds the number of DOFs, the MUSIC method cannot work.

\section{CONCLUSION}

In this letter, the difference coarray of moving platform based CLA proposed in [11] and [12] is analyzed, and the problem that the lags in the consecutive difference coarray part contribute negligibly to the increase of the DOFs after array motion is pointed out. To address this issue, an improved CLA configuration for moving platform is proposed. By judiciously designing the sensor element positions with two steps, all the lags contribute to fill holes after array motion, significantly lengthening the consecutive part of the difference coarray. Compared with the original CLA configuration, the proposed configuration has a difference coarray with much more consecutive lags. Therefore much more sources can be detected with the same number of sensor elements and same length of array motion. The moving platform based coprime arrays for two-dimensional DOA estimation [15] would be the future research orientation.

\section{REFERENCES}

[1] L. C. Godara, "Application of antenna arrays to mobile communications. II. beam-forming and direction-of-arrival considerations," Proceedings of the IEEE, vol. 85, no. 8, pp. 1195-1245, 1997.

[2] D. M. Vijayan and S. K. Menon, "Direction of arrival estimation in smart antenna for marine communication," in 2016 International Conference on Communication and Signal Processing (ICCSP). IEEE, 2016, pp. 1535-1540.

[3] P. Pal and P. P. Vaidyanathan, "Coprime sampling and the MUSIC algorithm," in 2011 Digital Signal Processing and Signal Processing Education Meeting (DSP/SPE). IEEE, 2011, pp. 289-294.

[4] J. Li, D. Jiang, and X. Zhang, "DOA estimation based on combined unitary ESPRIT for coprime MIMO radar," IEEE Communications Letters, vol. 21, no. 1, pp. 96-99, 2017.

[5] W. Zheng, X. Zhang, and H. Zhai, "Generalized coprime planar array geometry for 2-D DOA estimation," IEEE Communications Letters, vol. 21, no. 5, pp. 1075-1078, 2017.

[6] R. Schmidt, "Multiple emitter location and signal parameter estimation," IEEE Transactions on Antennas and Propagation, vol. 34, no. 3, pp. 276-280, 1986.

[7] R. Roy and T. Kailath, "ESPRIT-estimation of signal parameters via rotational invariance techniques," IEEE Transactions on Acoustics, Speech and Signal Processing, vol. 37, no. 7, pp. 984-995, 1989.

[8] S. Qin, Y. D. Zhang, and M. G. Amin, "Generalized coprime array configurations for direction-of-arrival estimation," IEEE Transactions on Signal Processing, vol. 63, no. 6, pp. 1377-1390, 2015.

[9] C.-L. Liu, P. Vaidyanathan, and P. Pal, "Coprime coarray interpolation for DOA estimation via nuclear norm minimization," in 2016 IEEE International Symposium on Circuits and Systems (ISCAS). IEEE, 2016, pp. 2639-2642.

[10] X. Wang and X. Wang, "Hole identification and filling in $k$-times extended co-prime arrays for highly efficient DOA estimation," IEEE Transactions on Signal Processing, vol. 67, no. 10, pp. 2693-2706, 2019.

[11] G. Qin, M. G. Amin, and Y. D. Zhang, "DOA estimation exploiting sparse array motions," IEEE Transactions on Signal Processing, vol. 67, no. 11, pp. 3013-3027, 2019.

[12] G. Qin, M. G. Amin, and Y. D. Zhang, "Analysis of coprime arrays on moving platform," in ICASSP 2019-2019 IEEE International Conference on Acoustics, Speech and Signal Processing (ICASSP). IEEE, 2019, pp. 4205-4209.

[13] G. Qin, Y. D. Zhang, and M. G. Amin, "DOA estimation exploiting moving dilated nested arrays," IEEE Signal Processing Letters, vol. 26, no. 3, pp. 490-494, 2019.

[14] S. Stergiopoulos and E. J. Sullivan, "Extended towed array processing by an overlap correlator,' The Journal of the Acoustical Society of America, vol. 86, no. 1, pp. 158-171, 1989.

[15] A. M. Elbir, "Two-dimensional DOA estimation via shifted sparse arrays with higher degrees of freedom," Circuits, Systems, and Signal Processing, vol. 38, no. 12, pp. 5549-5575, 2019. 